\title{
Efficient and Adaptive Node Selection for Target Tracking in Wireless Sensor Network
}

\author{
Juan Feng, Hongwei Zhao, and Baowang Lian \\ School of Electronic Information, Northwestern Polytechnical University, Xian 710072, China \\ Correspondence should be addressed to Juan Feng; fengjuankh@hotmail.com
}

Received 24 September 2014; Revised 9 May 2015; Accepted 20 May 2015

Academic Editor: Chi Chiu Chan

Copyright (C) 2016 Juan Feng et al. This is an open access article distributed under the Creative Commons Attribution License, which permits unrestricted use, distribution, and reproduction in any medium, provided the original work is properly cited.

In target tracking wireless sensor network, choosing the proper working nodes can not only minimize the number of active nodes, but also satisfy the tracking reliability requirement. However, most existing works focus on selecting sensor nodes which are the nearest to the target for tracking missions and they did not consider the correlation of the location of the sensor nodes so that these approaches can not meet all the goals of the network. This work proposes an efficient and adaptive node selection approach for tracking a target in a distributed wireless sensor network. The proposed approach combines the distance-based node selection strategy and particle filter prediction considering the spatial correlation of the different sensing nodes. Moreover, a joint distance weighted measurement is proposed to estimate the information utility of sensing nodes. Experimental results show that EANS outperformed the state-of-the-art approaches by reducing the energy cost and computational complexity as well as guaranteeing the tracking accuracy.

\section{Introduction}

Wireless Sensor Networks (WSNs) consist of a large amount of small, low-cost, and wirelessly connected sensor nodes deployed in an unattended natural environment. Since the sensor nodes are usually battery-powered and it is infeasible to replenish energy via replacing their battery after deployment, therefore optimization of energy consumption is essential in all aspects of WSN to prolong the network lifetime.

In WSNs, an important application of target tracking has received significant attention in recent years [1]. In this application, the sensor nodes collectively monitor the roaming path of moving objects in the area of deployment. Nevertheless, in target tracking, the user is only interested in the occurrence of a certain event, like movement of an intruder or enemy tanks in battle. Since sensor nodes are deployed densely in WSNs, a single target is recorded by many nodes while normally these records are spatially correlated. The degree of this correlation is inversely proportional to the distance among these nodes. In order to save energy, a smaller number of sensors in the event area rather than the whole number of the sensors are used to take the tracking tasks. Thus, dynamically choosing the best nodes for tracking task can balance the energy consumption of each sensor node and improve the lifetime of networks. Hence, it is crucial to select the optimization set of sensor nodes with the minimum cost and quality tracking performance. To solve the node selection problem, entropy-based information utility measurements were proposed, which are implemented with Bayesian Filter, such as [2]. Although they achieve good tracking accuracy, these methods are computationally expensive. In [3], the authors propose a weighted distancebased information utility measurement which needs less computation but can reach competitive tracking accuracy. Unfortunately, this method does not consider the spatial correlation of the sensed reports by different sensors. So far, the existing works can not meet all the goals of the target tracking WSNs.

With these motivations, we propose an efficient and adaptive node selection (EANS) strategy to dynamically choose the best set of sensor nodes for target tracking in WSNs. EANS combines the distance-based node selection strategy and particle filter prediction. The major objective of EANS is to keep reliable object tracking with minimum 
energy consumption. More precisely, the main contributions of this paper include the following:

(i) This paper proposed a novel spatial-correlated node selection strategy, called EANS, which selects the node with more residual energy and considers the spatial correlation of the sensors located at the different positions within the sensing range. Thus, EANS can balance energy consumption and guarantee the tracking reliability with the optimal set of sensor nodes and minimize working nodes so as to decrease the energy consumption significantly.

(ii) This paper proposed a joint distance weighted information utility measurement, in which the joint information utility can be presented as the overlap area of the sight lines of the possible sensors and the covariance-related ellipses. In this way, EANS evaluates the usefulness of a sensor node's observation without the complex entropy calculation and the a posteriori distribution estimation. Therefore, EANS can reduce the computational complexity and save the computational cost.

(iii) EANS considers not only the virtual range between sensors and target, but also the parallel degree of sensor's sight line to the target. In other words, it also considers the effect of angular diversity of sight lines so that the sensing range of the nodes in EANS is more reliable.

The rest of this paper is organized as follows: Section 2 gives an overview of related work. Then, the proposed efficient and adaptive node selection approach is presented in detail in Section 3. The experimental results are shown in Section 4. Finally, we conclude the paper in Section 5.

\section{Related Works}

Recently, the problem of selecting the best nodes for tracking a target in distributed WSNs has been attracting much research attention.

The simplest approach (such as [4]) is selecting the closest nodes which have the shortest distance to the target for tracking mission. This kind of methods has simple calculations but low tracking accuracy. To improve the tracking accuracy, a combination of the distance and utility function was proposed in [5], in which each node extracts a priority value based on its utility function, which is related to the distances of targets from that node. Nodes with less priority reduce their sensing range before their neighbors. Then, nodes that cannot cover any target or whose nearby targets are covered by neighbor nodes are not assigned the task and they are turned off. However, the approach required the location information of all the nodes.

In [6], the authors proposed a decentralized estimation method, which only needs to use local node information to achieve node selection. Furthermore, the number of active sensors is adaptively determined based on the absolute local innovations vector in [6]. In [7], posterior Cramer-Rao lower bound (PCRLB) was used as a criterion for sensor selection and exhaustive enumeration was adopted to search all possible combinations to seek for the minimum value of PCRLB. Unfortunately, the enumeration search would be a huge computational burden even when the density of a sensor network is just medium. In order to reduce complexity, the conditional posterior Cramer-Rao lower bound (C-PCRLB) in [8] was proposed as a sensor selection metric, which had a constraint on the total number of selected sensors to observe the target over a time window. These methods take the number of the selected nodes into account, but they did not consider the residual energy of each node and did not consider which nodes are more proper for the tracking mission. Moreover, there was also large computational burden.

In [9], the authors proposed a node selection scheme within the framework of particle filter, which uses clustering network architecture for collaborative tracking. This work considered the remaining energy of sensor node and achieved energy saving efficiently as well as required tracking reliability. However, the cluster head always consumes extra energy because of controlling the tracking. And the node selection also neglects the angular diversity of nodes. In [10], an energy-efficient node selection algorithm for bearingsonly sensors was proposed. The residual energy of a node was incorporated into the objective function to make a new criterion for node selection. However, all nodes in the paper needed time synchronized, which was difficult to implement in practice. In [11], a user selection scheme is proposed to minimize the overhead energy consumed by cooperative spectrum sensing. This method can conserve energy and achieve reasonably sensing accuracy, but it only focuses on the sensor node with a cognitive radio.

In [2], an entropy-based sensor selection heuristic approach is proposed. It is implemented with Bayesian Filter. The main idea of entropy-based approaches is to optimize an information utility function using the defined metrics. In [12], a mutual-information based sensor selection (MISS) algorithm was implemented for involvement in the mixture procedure. MISS allowed the sensor nodes with the highest mutual information about the target state to transfer data so that the energy ingestion was reduced while the preferred target position estimation accuracy was met. In [13], the authors proposed a light-weight sensing node selection scheme, which solved the sensing node selection problem by adopting a composite function in which information utility measure and energy consumption measure carry different weights. Although these approaches achieve good tracking accuracy, these methods all take the information utility brought by nodes as the objective function and have high computational complexity and expensive cost on computation.

In [14], the node selection is formulated as a subset selection problem which is shown to have a complexity that is NP-hard. At each step of the tracking task, the active nodes are selected from all sensors within the sensing range to minimize residue energy variations. In [15], the authors proposed a Fixed-Tree Relaxation-Based Algorithm (FTRA) and a very efficient Iterative Distributed Algorithm (IDA) to jointly optimize both sensor selection and routing structure and obtain the best possible estimation performance at a given 
querying node, for a given total power budget. However, these methods selected one sensor node for the tasks at each step and did not consider the locations correlations of the sensing nodes. Because the different combinations of the nodes within the sensing range can obtain the different tracking performance and different residue energy variations, the node selection problem should be considered completely. Besides, in [3], the authors proposed a weighted distancebased node selection method for bearings-only sensors in WSN. The sensor with the minimum weighted distance is activated for tracking mission. Although weighted distance method needs less computation comparing with entropybased sensor selection method, it chooses only one sensor to track the target each time and does not consider the spatial correlations of sensor nodes.

\section{Efficient and Adaptive Node Selection}

3.1. Network Model. In this paper, we consider a static WSN which is composed of one sink and $p$ randomly distributed sensor nodes $n_{i}, i \in[1, p]$ in a two-dimensional sensing field, where $p$ is the number of the deployed nodes. Our sensor network model has the following properties and assumptions:

(i) The sink is fixed and has an infinite power supply. And it gathers the information sensed by sensor nodes.

(ii) The distribution of sensor nodes is mutually independent. Every node is homogeneous and energy constrained.

(iii) Each node knows its position by using GPS or any localization algorithm. Let $X_{i}\left(x_{i}, y_{i}\right)$ be the location of node $n_{i}$.

Sensor nodes have three states, that is, active, sleep, and idle state. They remain in the sleep state most of the time and switch to the active state at specified time slots scheduled by the sink. In the active time slots, sensor nodes receive the assignment messages from the sink and check if there are sensing or relaying tasks in the next time instant. If there are tasks, they will keep active; otherwise they will sleep in next sensing instant. Afterward, the sink node predicts the next position of target by received data using particle filter algorithm. Then, the sink chooses the best nodes for the next task according to the joint distance weighted information utility measurement.

The goal of our work is to select the best subset of nodes for the next tracking task. Moreover, we decide which subset of nodes is the best according to two factors: (1) a joint distance weighted information utility and (2) the successful detection probability and residual energy of the candidate nodes. Therefore, given any time $t$, when a target's location $L_{t}=(x, y)$, the target can be reliably detected with less and balanced energy consumption.

3.2. Prediction Target Position Based on Particle Filter. In target tracking research field, particle filter (PF) has become a very effective algorithm because of its potential of coping with difficult nonlinear or non-Gaussian problems. PF with parallel structure is based on Monte Carlo simulation and

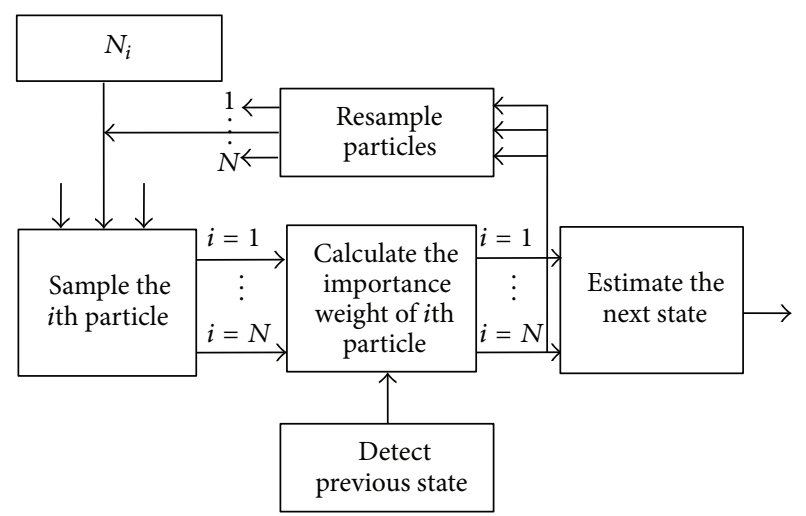

FIGURE 1: Schematic diagram of particle filter algorithm.

Bayesian sampling estimation theories [16]. And it is a sequential importance sampling method which is flexible and easy to be implemented.

As the sink node can obtain the collaborative sensing result of target positions, the PF algorithm is performed on the sink node to predict the target position in the next sensing instant. The schematic diagram of PF algorithm is shown in Figure 1.

The steps of PF are outlined as follows.

(1) Initialization. Assuming the initial target position probability distribution is $p(X(0))$, the particle set is shown as follows:

$$
\left\{X_{i}(0), i=1, \ldots, N_{s}\right\} \sim p(X(0))
$$

where $N_{s}$ is the number of particles. $X(0)$ is the target position estimation in the initial sensing instant. Thus, $X_{i}(k)=$ $\left[x_{i}(k), y_{i}(k)\right]$ is the estimated target position by particle $i$. In addition, the initial importance weight of particle $i$ is set as

$$
w_{i}(0)=\frac{1}{N_{s}} .
$$

(2) Iterations. The importance weight of $k+1$ time instant is calculated as follows:

$$
\begin{aligned}
w_{k+1}^{i} & =w_{k}^{i} \frac{p\left(Z_{k+1} \mid X_{k+1}^{i}\right) p\left(X_{k+1}^{i} \mid X_{k}^{i}\right)}{q\left(X_{k+1}^{i} \mid X_{k}^{i}, Z_{k+1}\right)} \\
& =w_{k}^{i} p\left(Z_{k+1} \mid X_{k+1}^{i}\right),
\end{aligned}
$$

where $Z_{k+1}$ is the observation of target position in the $t+1$ sensing instant.

(3) Resampling. When the variance of the importance weights becomes excessive, the particle needs to be resampled. The effective sample size is defined as

$$
N_{\text {eff }}=\frac{N_{s}}{1+\operatorname{Var}\left(w_{i}(k)\right)},
$$


- Sleep sensor node

- Active node
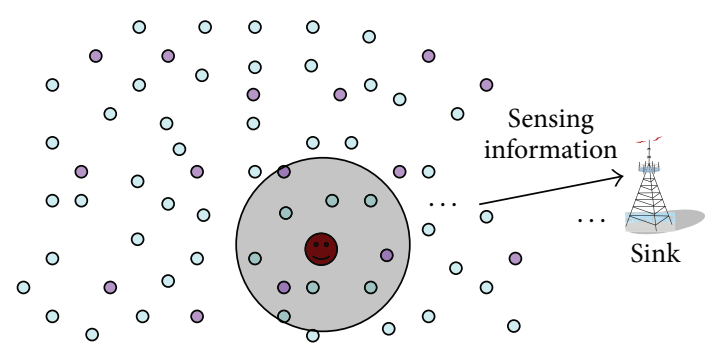

FIgURE 2: Node selection in target tracking WSNs.

where Var is the variance function. When $N_{\text {eff }}$ drops below threshold $N_{\text {th }}$, resample $N_{s}$ samples according to $p\left(X_{i}(k)\right.$ | $Z(k))$ and set importance weight of particle $i$ as

$$
w_{i}(k)=\frac{1}{N_{s}}
$$

Thus, the state of target position is updated as

$$
X(k+1)=\sum_{i=1}^{N_{s}} X_{i}(k+1) w_{i}(k+1) .
$$

In each sensing instant, the sink node can obtain a prior state of target position for the next sensing instant.

3.3. Efficient and Adaptive Node Selection. Dynamically choosing the best set of sensor nodes for tracking task can reduce the energy consumption of the network and improve tracking accuracy. As Figure 2 shows, there are many sensor nodes in the sensing area. However, tracking a target does not need so many sensor nodes. Generally, we should select sensor nodes which can bring more information among the candidate nodes in this area. The sink is required to decide which nodes should be active for the next task and which nodes should be kept sleeping to save energy.

In the entropy-based method, the entropy is used as information utility measure. The information utility of node $N_{i}$ is calculated by

$$
\begin{aligned}
\operatorname{Utility}\left(N_{i}\right) & =-H p\left(X_{t+1} \mid \bar{Z}_{t+1}\right) \\
& =\sum P\left(X_{t+1} \mid \bar{Z}_{t+1}\right) \log P\left(X_{t+1} \mid \bar{Z}_{t+1}\right),
\end{aligned}
$$

where $P\left(X_{t+1} \mid \bar{Z}_{t+1}\right)$ is the posterior distribution of the target's state. More details of entropy-based information utility measure can be found in [2].

According to Section 3.2, we have the knowledge of target state predicted by PF algorithm. From that, we can obtain the predicted location of the target as $\left(\bar{x}^{\prime}, \bar{y}^{\prime}\right)$ and a covariance matrix as

$$
\operatorname{Cov}^{\prime}=\left[\begin{array}{cc}
D_{x^{\prime}}^{2} & D_{x^{\prime}} D_{y^{\prime}} r \\
D_{x^{\prime}} D_{y^{\prime}} r & D_{y^{\prime}}^{2}
\end{array}\right],
$$

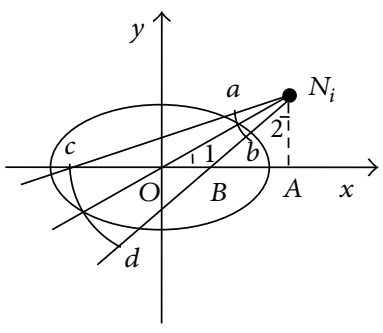

FIGURE 3: Illustration of information utility measure in weighted distance method.

where $D_{x^{\prime}}$ and $D_{y^{\prime}}$ are deviations along $x^{\prime}$ - and $y^{\prime}$-axes, respectively, and $r$ is the correlation coefficient. Then a new coordinate system, whose origin is at $\left(\bar{x}^{\prime}, \bar{y}^{\prime}\right)$ and whose axes are along the direction of the eigenvectors of $\mathrm{Cov}^{\prime}$, can be established [3]. In the new coordinate system, the predicted belief is represented by zero-mean Gaussian density function with covariance:

$$
\operatorname{Cov}=\left[\begin{array}{cc}
\sigma_{x}^{2} & 0 \\
0 & \sigma_{y}^{2}
\end{array}\right],
$$

where $\sigma_{x}^{2}$ and $\sigma_{y}^{2}$ are the largest and smallest eigenvalue of $\mathrm{Cov}^{\prime}$, respectively. Then, the state uncertainty of the target can be represented by an ellipse whose major axis and minor axis are $3 \sigma_{x}^{2}$ and $3 \sigma_{y}^{2}$, respectively, as Figure 3 shows. Choosing 3 sigma is because, within the region covered by a 3 sigma ellipse, the target, whose state follows a Gaussian distribution, will appear by the chance of $98.89 \%$ [17].

Assuming the measurement error $\Delta \delta_{i}$ is known, the location $\left(x_{i}, y_{i}\right)$ of node $N_{i}$ can be denoted by the polar coordinates $\left(\delta_{i}, R_{i}\right)$. Then, the information utility in weighted distance method is defined as

$$
\operatorname{Utility}\left(N_{i}\right)=- \text { Area }_{a b c d} \text {. }
$$

That is, the information utility in the weighted distance method can be approximated as the intersected area of the sight lines of a candidate node and the ellipse, as Figure 3 shows. The smaller the area is, the more the information is provided by the node.

Unfortunately, because the locations of sensing nodes can affect each other on the estimation of target's state and position, just considering one sensor node each time is not enough when node selecting algorithm is implemented. As shown in Figure 4, nodes $N_{i}, N_{j}$, and $N_{s}$ are three of candidate sensing nodes. The area enclosed by nodes $N_{i}$ and $N_{j}$ (Figure 4(a)) is much larger than the area enclosed by nodes $N_{i}$ and $N_{s}$ (Figure 4(b)). That is, if we both choose two sensor nodes to track, choosing nodes $N_{i}$ and $N_{s}$ can obtain more certainty of the target's state than that of choosing nodes $N_{i}$ and $N_{j}$. Therefore, when nodes are selected, the correlation of location of sensor nodes should be considered. And we proposed a joint distance weighted information utility measurement to effectively select nodes for tracking mission. It can be represented as follows:

$$
\text { Joint_utility }\left(N_{i}, N_{j}\right)=-\left(C \_A_{r e a} \cap C_{-} A_{r e a}\right) \text {, }
$$




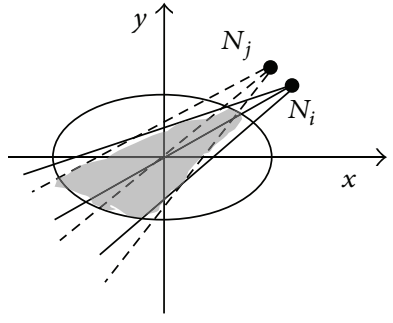

(a)

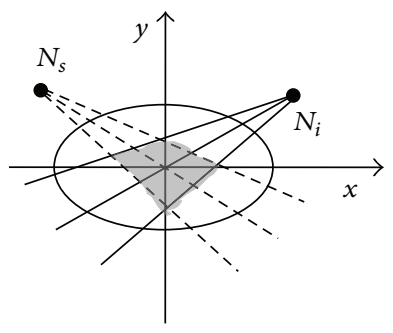

(b)
FIGURE 4: Effect of node's location on information utility measure.

where $C_{-}$Area $_{N i}$ is the certainty area enclosed by the sight lines of node $N_{i}$ and the ellipse (such as Figure 3). Consider $C_{-}$Area $_{N i} \cap C_{-}$Area $_{N j}$ is the overlapped area of the certainty area of nodes $N_{i}$ and $N_{j}$. The smaller the area $C_{-} A_{\text {Area }}{ }_{N i} \cap$ $C_{-}$Area $_{N j}$ is, the more the certainty by using the node's information will be there.

The equation of the uncertainty ellipse is known as

$$
\frac{x^{2}}{\left(3 \sigma_{x}\right)^{2}}+\frac{y^{2}}{\left(3 \sigma_{y}\right)^{2}}=1 \text {. }
$$

In order to obtain the joint distance weighted information utility, we just need to calculate the equation of the sight lines of the sensor node. The process of calculation is as follows (taking Figure 3 as an example).

(1) The line crossed node $N_{i}$ and the origin is represented as

$$
\frac{x}{x_{i}}=\frac{y}{y_{i}}
$$

Thus, the distance from node $N_{i}$ to the origin is

$$
\sqrt{x_{i}^{2}+y_{i}^{2}}
$$

(2) Calculating the degree of the angle $\angle 1$ is

$$
\angle 1=\arcsin \left(\frac{y_{i}}{\sqrt{x_{i}^{2}+y_{i}^{2}}}\right) .
$$

(3) Calculating the distance from node $B$ to node $A$ is

$$
d_{B A}=\tan \left(\frac{\pi}{2}-\theta-\arcsin \left(\frac{y_{i}}{\sqrt{x_{i}^{2}+y_{i}^{2}}}\right)\right) y_{i},
$$

where $\theta$ is the angle of sight lines of node $N_{i}$. Therefore, we get $d_{\mathrm{OB}}=x_{i}-d_{B A}$ and the line equation of $B N_{i}$ is obtained. Accordingly, we can get equations of the other line and calculate the certainty area. For simplicity, $C A_{-} \operatorname{Area}_{N i} \cap$ $C_{-}$Area $_{N j}$ can be approximately calculated as the number of the valid points. Suppose $n_{a}$ points are generated in the ellipse evenly and randomly; then the fallen points in the area of C_Area ${ }_{N i} \cap C_{-}$Area $_{N j}$ are called valid points. In this way, the complex entropy calculation is converted to the simple comparing operation. Moreover, we can control the computational complexity and accuracy by choosing proper $n_{a}$. In our work, we set $n_{a}=10^{3}$.

When the sink obtains the estimated position of the target $\left(\bar{x}^{\prime}, \bar{y}^{\prime}\right)$, it needs to select and awake the sensor nodes to track for next time instant. All the nodes whose sensed range includes $\left(\bar{x}^{\prime}, \bar{y}^{\prime}\right)$ are regarded as candidate nodes and the corresponding area is called the sensing area. The sink first chooses one node $N_{f}$ which has the most residual energy in the sensing area. Then, the joint information utility of $N_{f}$ and the other candidate nodes is calculated, respectively. And choose the node which has the largest joint information utility as the tracking node. This procedure is continued until enough tracking nodes are selected. It can be depicted in Algorithm 1.

\section{Performance Evaluation}

To evaluate the performance of the proposed approach of EANS, we simulated a network with 100 sensors nodes randomly laid out in a $100 \mathrm{~m} \times 100 \mathrm{~m}$ area. We also compare our simulation results with the closest node selection (in which the nodes closest to the target are selected to track), the weighted distance node selection method (in which a node is selected once time according to its own information utility) and entropy-based method in terms of Mean square errors, execution time and energy cost.

The sink is fixed and located at $(100,150)$. The sensing range of each sensor node is $R_{\text {sensing }}=10 \mathrm{~m}$. Moreover, each sensor has a sight angle of 40 degrees and the measurement error follows a Gaussian distribution whose standard deviation is 3 degrees. We also assume that each node has an initial energy of $1 \mathrm{~J}$ (Joule). A very widely used energy consumption model (such as the one in [18]) is adopted, as described in

$$
\begin{aligned}
E_{T x}(k, d) & =\left(E_{T x \text {-elec }}+\varepsilon_{\text {amp }} * d^{\alpha}\right) * k, \\
E_{R x}(k) & =E_{R x \text {-elec }} * k
\end{aligned}
$$

where $E_{T x \text {-elec }}$ and $E_{R x \text {-elec }}$ are the energy consumption of the transmitter and receiver electronics. $\varepsilon_{\text {amp }}\left[\right.$ Joule $/\left(\right.$ bit $\left.\left.\cdot \mathrm{m}^{\alpha}\right)\right]$ is a constant that represents the energy needed to transmit one bit to achieve an acceptable signal to noise ratio over distance $d$, and $\alpha$ is the path loss exponent $(2 \leq \alpha \leq 5)$ which depends on the channel quality. We can assume $E_{T x \text {-elec }}=E_{R x \text {-elec }}=E_{\text {elec }}$ and set parameters $E_{\text {elec }}=50 \mathrm{~nJ} / \mathrm{b}, \varepsilon_{\mathrm{amp}}=100 \mathrm{pJ} /\left(\mathrm{b} \cdot \mathrm{m}^{2}\right)$, and $\alpha=2$. The power consumption of radio in idle and sleep mode is $0.22 \mathrm{~mW}$ and $0.000006 \mathrm{~mW}$, respectively. The bandwidth of wireless channel is $1 \mathrm{Mbps}$ and we adopted the MAC model of IEEE 802.15.4. In our experiments, a data packet size is 2000 bits and a control message is 64 bits long. In PF algorithm, the particle number $N_{s}$ is set as 200 and the resampling threshold $N_{\text {th }}=0.2 N_{s}$. The sampling period is $1 \mathrm{~s}$.

For a moving target, we assume a target in the sensing area moves randomly with maximum acceleration $a_{\max }=2 \mathrm{~m} / \mathrm{s}^{2}$ 


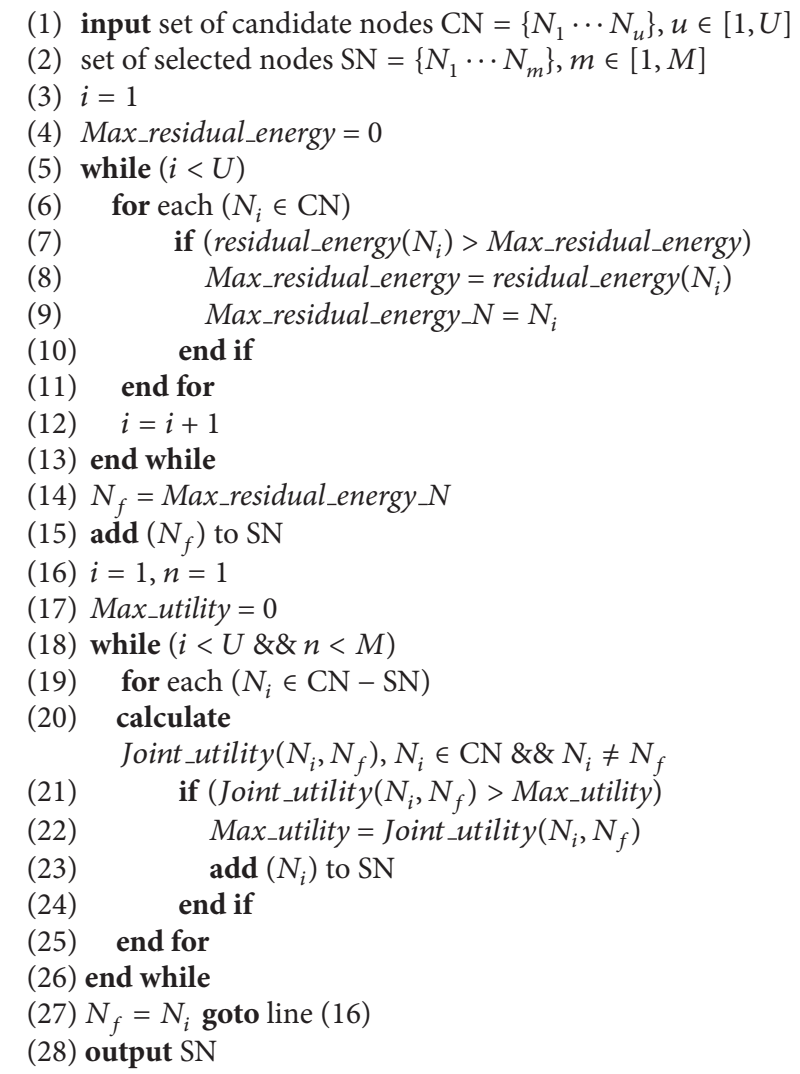

Algorithm 1: EANS node selection algorithm.

and maximum velocity $v_{\max }=6 \mathrm{~m} / \mathrm{s}$. This process model is given by

$$
X_{k+1}=\left[\begin{array}{cccc}
1 & T & 0 & 0 \\
0 & 1 & 0 & 0 \\
0 & 0 & 1 & T \\
0 & 0 & 0 & 1
\end{array}\right] X_{k}+\left[\begin{array}{c}
T^{2} \\
2 \\
T \\
\frac{T^{2}}{2} \\
T
\end{array}\right] v_{k}
$$

where $X_{k}=\left[x, v_{x}, y, v_{y}\right]^{T}$ is the state of the moving target at $k$ time instant. $T$ is the sampling time interval, $T=1 \mathrm{~s}$. $x, v_{x}$ are the position and velocity of the target in the direction of $x$-axis, respectively. $y, v_{y}$ are the position and velocity of the target in the direction of $y$-axis, respectively. $v_{k}$ is the state transition noise of the target: $v_{k} \sim N(0, \sigma), \sigma=2^{\circ}$. In addition, the observation model is

$$
\begin{gathered}
Z_{k+1}=H X_{k+1}+w_{k+1}, \\
H=\left[\begin{array}{llll}
1 & 0 & 0 & 0 \\
0 & 1 & 0 & 0 \\
0 & 0 & 1 & 0 \\
0 & 0 & 0 & 1
\end{array}\right],
\end{gathered}
$$

where $H$ is the observation matrix. $w_{k+1}$ is the measurement noise which is assumed to be white Gaussian noise sequence with zero means and the variance $\sigma$. The target trajectory is generated as shown in Figure 5 and this scenario can represent the generalization of the target tracking problem. For each simulation, we run at least 50 times with different random node distributions and the average results are shown in Figure 6.

Figure 6 shows the mean square positioning errors in the different approaches when 3 nodes are selected for tracking. From Figure 6, we can see that the closest node selection method gives the biggest errors because it does not consider the moving trend of the target and the effect of the angular diversities of sensor nodes. The entropy-based method has the most accurate results because it executes a lot of probability predictions and entropy calculations which are computationally intensive. The weighted distance node selection method has less accuracy than that in the proposed EANS because it selects one node each time and did not consider the relations among the locations of the sensor nodes. As shown in Figure 6, EANS method achieves almost the same precision as entropy-based approach because it adopts the joint distance weighted information utility measurement considering the spatial correlation of sensor nodes to provide much more useful information for node selections. 


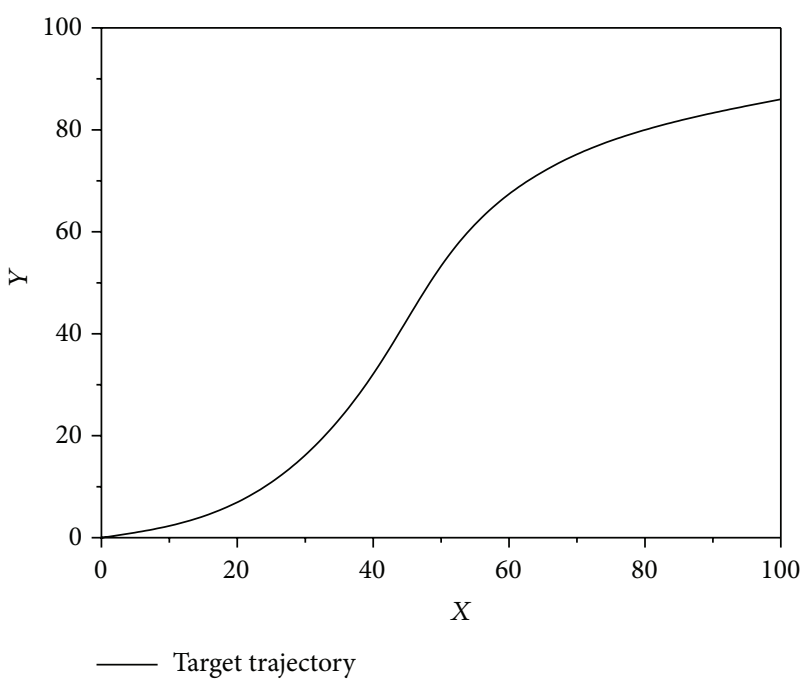

FIGURE 5: Target trajectory in the sensing area.

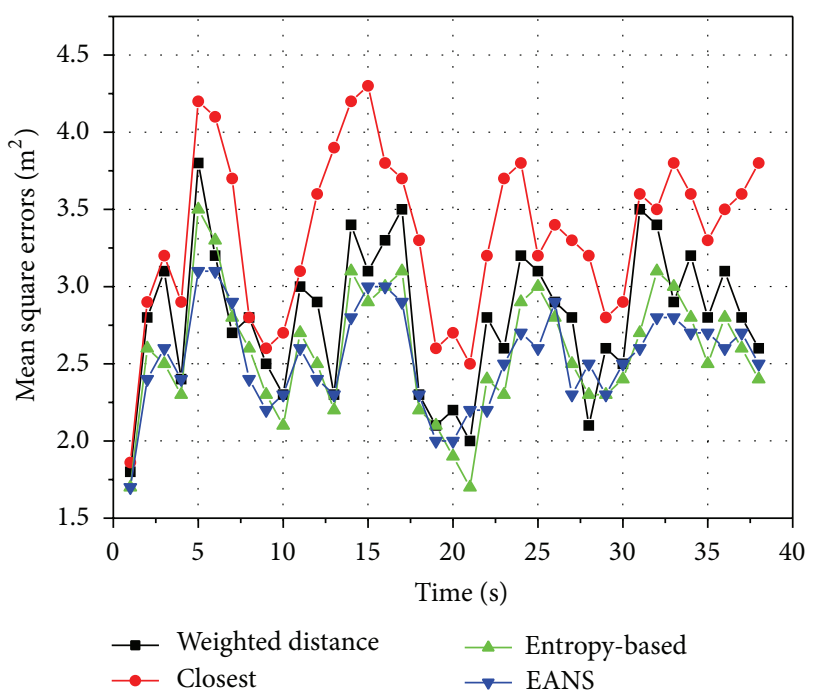

FIGURE 6: Mean square positioning errors in different methods.

In order to investigate the computational complexities of these approaches in the simulations, the experiments were carried out keeping the other parameters fixed and progressively increasing the number of active candidate nodes to be selected for tracking in the sensing area. Specifically, the candidate nodes increase from 1 to 10. Figure 7 shows the execution time of the different approaches. Not surprisingly, all the algorithms use more execution time with increasing the number of candidate nodes. This is because more computations are needed for more candidate sensor nodes. The execution time of the entropy-based approach quickly rises in pace with the increasing candidate nodes and it is the largest among these approaches because more computation of predictions and mutual information utilities is needed and it is more complex with a large number of sensor nodes. The closest approach achieves the best results compared to the others because it is simple to estimate the distance

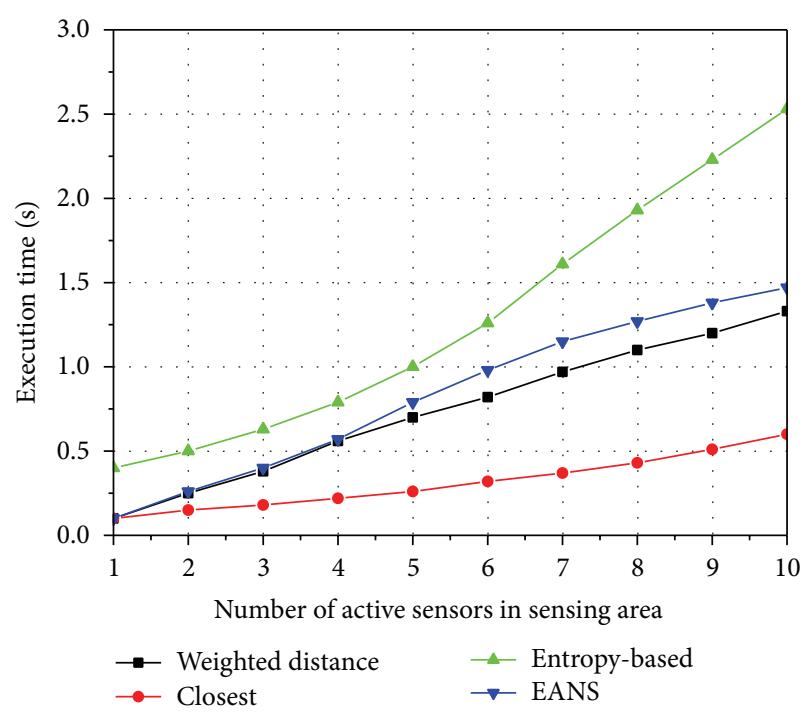

FIGURE 7: Comparison of execution time versus the number of active sensors in the sensing area.

TABLE 1: Comparison of different approaches of average execution time.

\begin{tabular}{lcccc}
\hline & \multicolumn{4}{c}{ Sensing ranges } \\
Terms & \multicolumn{4}{c}{ Sensing range of sensors } \\
& $10 \mathrm{~m}$ & $20 \mathrm{~m}$ & $40 \mathrm{~m}$ & $80 \mathrm{~m}$ \\
\hline $\begin{array}{l}\text { Average number of } \\
\text { nodes within sensing } \\
\text { range }\end{array}$ & 4 & 9 & 28 & 76 \\
$\begin{array}{l}\text { Average execution time } \\
\text { of node selection (s) }\end{array}$ & & & & \\
$\quad$ Weighted distance & 0.535 & 1.168 & 3.116 & 6.389 \\
$\quad$ Closest & 0.115 & 0.492 & 0.834 & 2.529 \\
$\quad$ Entropy-based & 0.763 & 2.218 & 6.273 & 13.517 \\
$\quad$ EANS & 0.563 & 1.272 & 3.656 & 7.395 \\
\hline
\end{tabular}

between the node and target. The execution time of EANS is a little larger than that of the weighted distance approach when candidate nodes increase because more calculations are needed for the joint information utility. However, the results of EANS are very near to that of the weighted distance approach and the largest difference in these two approaches is just $0.1 \mathrm{~s}$. From Figures 6 and 7, we can see that EANS has relative lower computational complexity to achieve more accuracy.

Moreover, nowadays, sensor nodes are available with a longer or variable sensing range. If sensing ranges of sensors are changed, the number of sensor nodes within the sensing range is also changed. Table 1 shows the average execution time of node selection varying with the different sensing range in the approaches. Obviously, the longer sensing range the sensors have, the more nodes are within sensing range; that is to say, the node selection algorithms need to select the working nodes from the large number of nodes. Therefore, the average execution time of each approach is increased. 


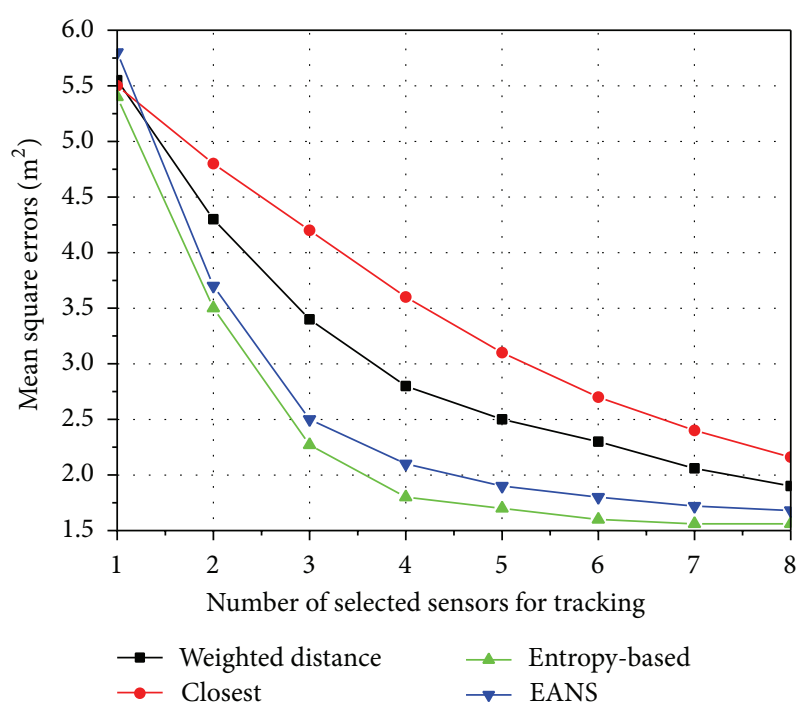

FIgURE 8: Mean square positioning errors versus the number of selected nodes.

Nevertheless, our EANS also have good performance. With regard to tracking accuracy, the increased sensing ranges of nodes have no effects on it, because the tracking accuracy depends on the selected working nodes and the number of selected working nodes.

Figure 8 shows the mean square positioning errors versus the number of selected nodes for tracking. Obviously, the more nodes are selected, the more accuracy can be obtained when the selected nodes are in the range of 1 to 6 . If the number of the selected nodes is bigger than 6 , the accuracy increases slowly and inconspicuously because some selected nodes bring the repetitive and useless information. As shown in Figure 8, although the accuracy of the closest method rises with the increasing selected nodes, it has the worst results because the selected nodes provide less useful information. When the selected nodes grow from 1 to 5 , the accuracy of EANS increases substantially because the most useful nodes set for tracking can be selected by EANS. The weighted distance approach has worse results than that in EANS because the correlations of the nodes are not considered in node selection. Comparing the results of EANS with that in the closest and weighted distance approaches, it is seen that using a fewer nodes for tracking in EANS can achieve the accuracy which is obtained by more selected nodes in the other two approaches. Therefore, EANS approach can reduce the number of the active nodes for tracking so as to save energy and guarantee the tracking performance.

Finally, Table 2 shows the comparison of the average energy cost of the system and fail tracking percentage in the four approaches. We can see that EANS has the least energy cost among these approaches. This is caused by the following reasons: (1) EANS reduces the number of active nodes for tracking tasks. (2) The target state prediction in EANS is implemented at the sink, which does not spend the energy of sensor nodes. (3) It has relative less computational works. Although the closest approach has the simplest calculations,
TABLE 2: Comparison of different approaches for tracking.

\begin{tabular}{lcc}
\hline Approaches & \multicolumn{2}{c}{ Terms } \\
& $\begin{array}{c}\text { Average energy cost } \\
\text { of the system }(\mathrm{J})\end{array}$ & $\begin{array}{c}\text { Fail tracking } \\
\text { percentage }(\%)\end{array}$ \\
\hline Weighted distance & 0.226 & 0.73 \\
Closest & 0.251 & 1.26 \\
Entropy-based & 0.243 & 0.63 \\
EANS & 0.213 & 0.66 \\
\hline
\end{tabular}

it needs more active nodes to guarantee the tracking accuracy so that it costs the most energy. The entropy-based approach costs more energy because it has massive calculating works. In addition, because the closest approach just selects the nodes near to the current location of the target and does not consider the next target location, it has higher fail tracking percentage than the others. To sum up, EANS achieves more energy efficiency and less computational complexity while degrading the tracking performance.

\section{Conclusions}

This paper proposed an efficient and adaptive node selection (EANS) approach for target tracking WSNs. EANS combines the distance-based node selection strategy and particle filter which is implemented at the sink to predict target states. The proposed spatial-correlated node selection algorithm uses a joint distance weighted measurement to estimate the information utility of sensing nodes. EANS has better performance than the other approaches by considering the spatial correlations of the sensing nodes in the process of node selections. The simulation results proved that EANS outperformed the state-of-the-art approaches by reducing the energy cost and computational complexity as well as guaranteeing the tracking accuracy.

\section{Conflict of Interests}

The authors declare that there is no conflict of interests regarding the publication of this paper.

\section{Acknowledgments}

This work was supported by the National Natural Science Foundation of China under Grant 61301094, the Aerospace Innovation Foundation of Shandong province under Grant 2014JJ009, and the Fundamental Research Funds for the Central Universities under Grant 3102015ZY040.

\section{References}

[1] S. Bhatti and J. Xu, "Survey of target tracking protocols using wireless sensor network," in Proceedings of the 5th International Conference on Wireless and Mobile Communications (ICWMC '09), pp. 110-115, IEEE, August 2009.

[2] H. Wang, G. Pottie, K. Yao, and D. Estrin, "Entropy-based sensor selection heuristic for target localization," in Proceedings 
of the 3rd International Symposium on Information Processing in Sensor Networks (IPSN '04), pp. 36-45, Berkeley, Calif, USA, April 2004.

[3] W. Zhao, Y. Han, H. Wu, and L. Zhang, "Weighted distance based sensor selection for target tracking in wireless sensor networks," IEEE Signal Processing Letters, vol. 16, no. 8, pp. 647650, 2009.

[4] J. Qian, X. Jin, and Z. Yu, "Energy-efficient node selection for acoustic source localization in wireless sensor network," in Proceedings of the 6th International Conference on Wireless Communications, Networking and Mobile Computing (WiCOM '10), pp. 1-4, Chengdu, China, September 2010.

[5] M. Naderan, M. Dehghan, and H. Pedram, "Sensing task assignment via sensor selection for maximum target coverage in WSNs," Journal of Network and Computer Applications, vol. 36, no. 1, pp. 262-273, 2013.

[6] M. Zoghi and M. H. Kahaei, "Adaptive sensor selection in wireless sensor networks for target tracking," IET Signal Processing, vol. 4, no. 5, pp. 530-536, 2010.

[7] F. R. Armaghani, I. Gondal, and J. Kamruzzaman, "Dynamic sensor selection for target tracking in wireless sensor networks," in Proceedings of the IEEE 74th Vehicular Technology Conference (VTC Fall '11), pp. 1-6, San Francisco, Calif, USA, September 2011.

[8] E. Masazade, R. Niu, and P. K. Varshney, "An approximate dynamic programming based non-myopic sensor selection method for target tracking," in Proceedings of the 46th Annual Conference on Information Sciences and Systems (CISS '12), pp. 1-6, March 2012.

[9] Y. Wang and D. Wang, "Energy-efficient node selection for target tracking in wireless sensor networks," International Journal of Distributed Sensor Networks, vol. 2013, Article ID 830950, 6 pages, 2013.

[10] X. Jiang, W. He, X. Yang, and Y. Wang, "An energy-efficient node selection algorithm in bearings-only target tracking sensor networks," International Journal of Distributed Sensor Networks, vol. 2014, Article ID 483279, 12 pages, 2014.

[11] N. U. Hasan, W. Ejaz, S. Lee, and H. S. Kim, "Knapsackbased energy-efficient node selection scheme for cooperative spectrum sensing in cognitive radio sensor networks," IET Communications, vol. 6, no. 17, pp. 2998-3005, 2012.

[12] B. Chithra and N. J. R. Muniraj, "A mutual information based sensor selection and information controlled transmission power adjustment," in Proceedings of the International Conference on Optical Imaging Sensor and Security (ICOSS '13), pp. 110, July 2013.

[13] H. Ma, "Sensing nodes selection scheme for distributive target tracking in wireless sensor networks," in Proceedings of the 37th Annual Conference of the IEEE Industrial Electronics Society (IECON '11), pp. 2164-2169, IEEE, Melbourne, VIC, Australia, November 2011.

[14] X. Q. Hu, Y. H. Hu, and B. G. Xu, "Energy-balanced scheduling for target tracking in wireless sensor networks," ACM Transactions on Sensor Networks, vol. 11, no. 1, article 21, 2014.

[15] S. Shah and B. Beferull-Lozano, "Joint sensor selection and multihop routing for distributed estimation in ad-hoc wireless sensor networks," IEEE Transactions on Signal Processing, vol. 61, no. 24, pp. 6355-6370, 2013.

[16] Y. Yu and Q. Cheng, "Particle filters for maneuvering target tracking problem," Signal Processing, vol. 86, no. 1, pp. 195-203, 2006.
[17] M. Abramowitz and I. A. Stegun, Handbook of Mathematical Functions, Dover, New York, NY, USA, 1965.

[18] Q. Mamun, S. Ramakrishnan, and B. Srinivasan, "Selecting member nodes in a chain oriented WSN," in Proceedings of the IEEE Wireless Communications and Networking Conference (WCNC '10), pp. 1-6, April 2010. 


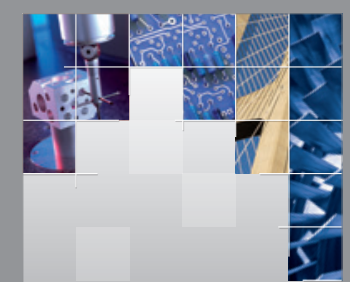

\section{Enfincering}
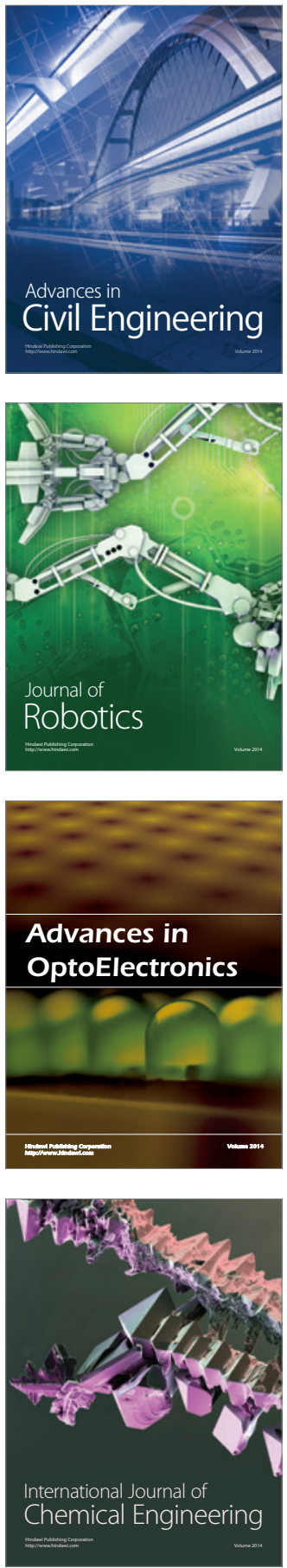

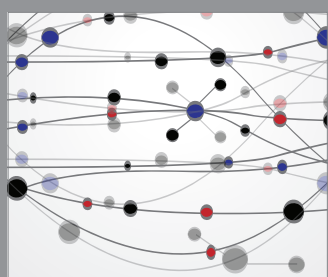

The Scientific World Journal

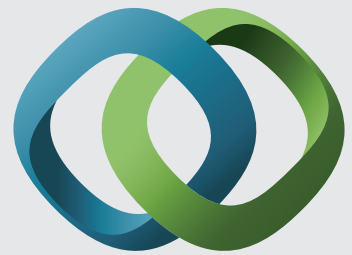

\section{Hindawi}

Submit your manuscripts at

http://www.hindawi.com
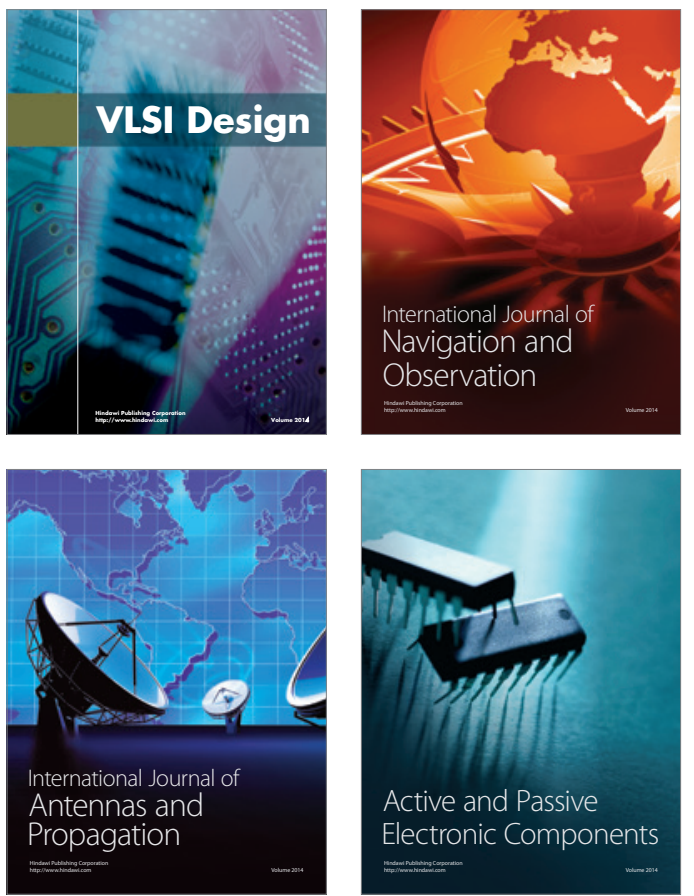
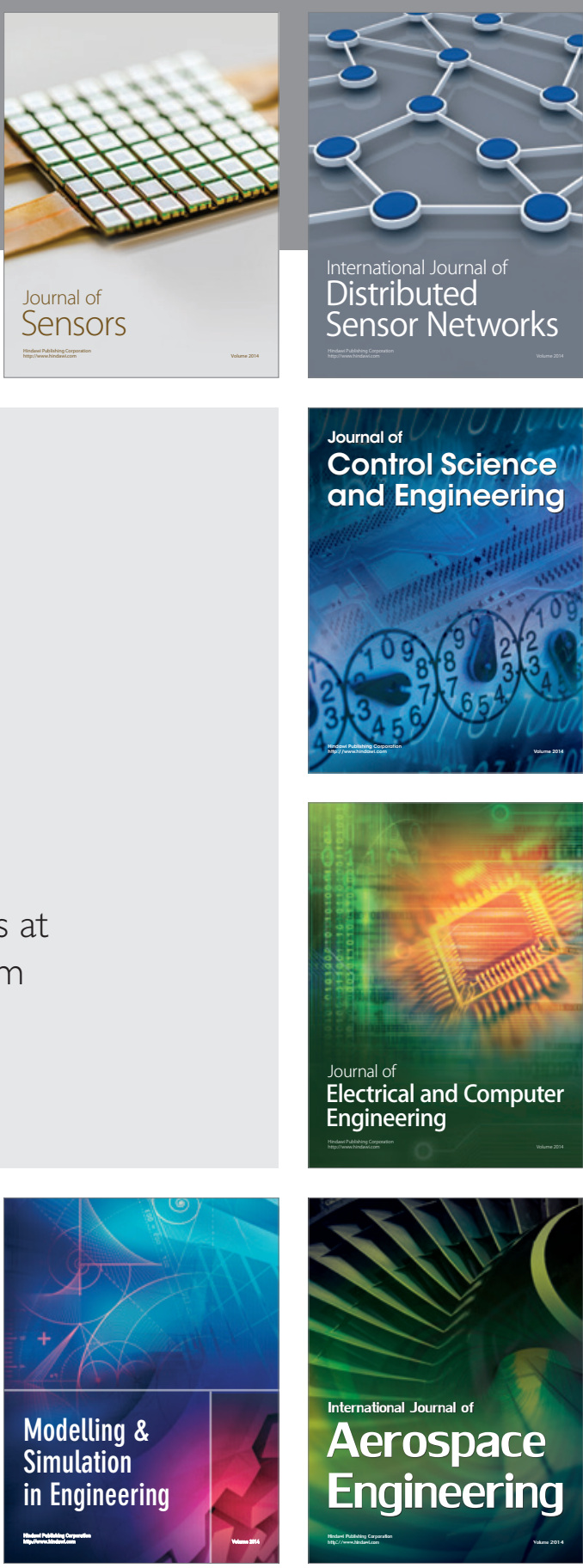

International Journal of

Distributed

Sensor Networks

Journal of

Control Science

and Engineering
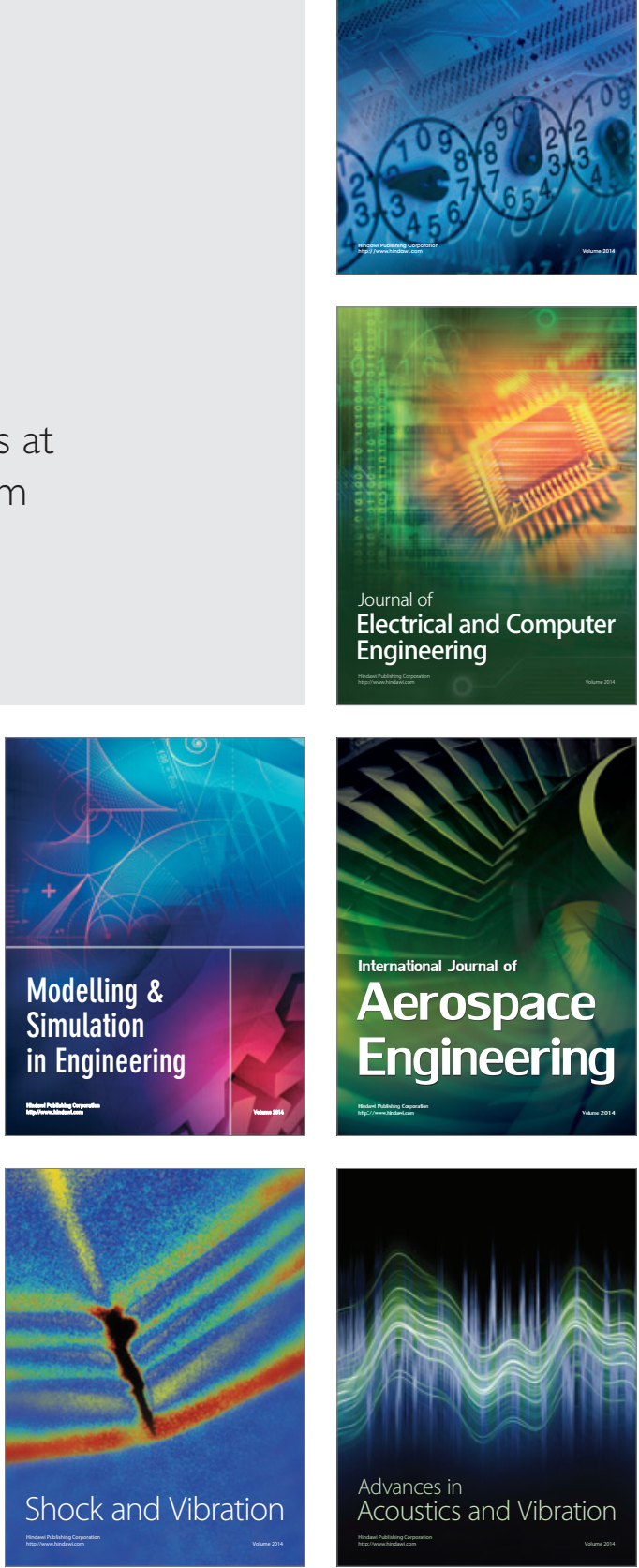